\title{
SEARCHING FOR $p$-ADIC EIGENFUNCTIONS
}

\author{
Fernando Q. GouvêA And Barry Mazur
}

To A. O. L. Atkin, on his retirement

In doing $p$-adic analysis on spaces of classical modular functions and forms, it is convenient and traditional to broaden the notion of "modular form" to a class called "overconvergent $p$-adic modular forms." Critical for the analysis of the $p$-adic Banach spaces composed of this wider class of forms is the "Atkin $U$-operator", which is completely continuous and whose spectral theory (still not very well understood) seems to be the key to a good deal of arithmetic. The part of the spectrum of $U$ corresponding to eigenvalues which are $p$-adic units ${ }^{1}$ is somewhat more understood, thanks to the work of Hida. As for the rest of the spectrum, it is surprising how fragmentary our information is (although recent work of Coleman, resolving in part some prior conjectures of ours, has improved the situation).

We have begun an experimental search for nonclassical (but "overconvergent") eigenfunctions in a fairly simple way. We take a number of classical modular functions which are $p$-adically overconvergent (e.g., $j$, $1 / j, \ldots)$, and try to find their $p$-adic " $U$-eigenfunction expansion." There is a straightforward computational procedure to approximate such eigenfunction expansions, even though, on a theoretical level, we do not even know that the "expansions" that our algorithm produces converge in any sense, or even settle down numerically. Experimentally, they seem to, and they produce candidate Fourier expansions.

In our computations, we specialize principally to $p=5$. The same eigenfunctions (produced by our algorithm) seem to occur as terms in each of the eigenfunction expansions we have calculated. This leads us to suspect that we have in fact encountered all the 5-adic eigenfunctions

1991 Mathematics Subject Classification. Primary 11F33.

Key words and phrases. p-adic modular functions, overconvergence, U operator.

Received February 16, 1995; revised September 8, 1995.

The first author was supported in part by grants DMS-9203469 and DMS-9401313 from the NSF.

${ }^{1}$ We call this the slope zero part, and in general define the slope of a generalized $U$-eigenform to be the $p$-adic valuation of its eigenvalue. 


\begin{tabular}{|c||c|c|c|c|c|c|}
\hline$p$ & 2 & 3 & 5 & 7 & 11 & 13 \\
\hline$j=$ & 0 & 0 & 0 & -1 & $0,-1$ & 5 \\
\hline
\end{tabular}

TABLE 1. Supersingular $j$-invariants for small primes

of small slope (i.e., slope at most 5) and tame level 1. More specifically, up to scaling, we found exactly one "candidate eigenfunction" of slope 0 (the constant function 1), one of slope 1, one of slope 4, and one of slope 5. See section 4 below for an approximation to the Fourier expansions (of the ones of positive slope!). A separate check on the consistency of these experimental "results," was provided by Jeremy Teitelbaum, who used Koike's formula in [13] to compute the characteristic series $\operatorname{det}(1-$ $\left.t U_{k}\right) \bmod 3^{27}$ for $p=3$ and $\bmod 5^{28}$ for $p=5$. His data is consistent with our findings, and suggests that there are five 3 -adic overconvergent eigenfunctions of slope $\leq 10$ (one each of slopes $0,2,6,8,10$ ) and there are six 5 -adic eigenfunctions of slope $\leq 9$ (one each of slopes $0,1,4,5,8$, 9). It might be of interest to study their associated Galois representations and $p$-adic $L$-functions.

We offer this article as a small retirement present to A. O. L. Atkin, whose pioneering work on these issues has been the inspiration for these investigations. In [1], [3], and [2], he describes his discovery "that some ' $p$-adic' theory of Hecke operators exists [which is] analogous to the exact classical theory" (see [2, p. 33]). In particular, he showed that iterating the $U$ operator on forms of weight zero would produce approximate eigenforms, very much as we have done. In the case of the $j$ function and $p=13$, Atkin has given a proof (see [3]) that this procedure yields a $p$-adic eigenform of slope 0 . He has written (see [1]) that his methods extend to prove that this process converges to a $p$-adic eigenform of slope 1 (the form we call $f_{1}$ below) for $p=5$ and similarly for $p=3$ and $p=2$. Our work therefore follows in his footsteps.

\section{Overconvergent modular functions}

Let $p$ be a prime number, and let $X=\mathbb{P}_{/ \mathbb{Z}_{p}}^{1}$, which we will think of as the " $j$-line," i.e., the modular curve of level 1 . The set of supersingular $j$-invariants in $X\left(\overline{\mathbb{F}}_{p}\right)$ is a finite set of points, all rational over $\mathbb{F}_{p^{2}}$, e.g., if $p \leq 5, j=0$ is the only supersingular value of $j$, and if $p=7, j=-1$ is the only supersingular value.

Table 1 lists these points for a few small primes $p$.

The notion of "overconvergent $p$-adic modular functions" (i.e., overconvergent modular forms of weight zero) was introduced by Katz in [12] 
(more information can also be found in [6], [9], and [7]). We give here a more "concrete" description of such functions, based on the idea that one can understand a $p$-adic object by understanding its reduction modulo $p^{\nu}$ for all $\nu \geq 1$.

Definition 1. An overconvergent p-adic modular function with integral $q$ expansion $f$ is a sequence of rational functions $f_{\nu}$ on the projective line $X_{\mathbb{Z} / p^{\nu} \mathbb{Z}}=X \otimes \mathbb{Z} / p^{\nu} \mathbb{Z}$ over $\mathbb{Z} / p^{\nu} \mathbb{Z}$ for $\nu=1,2, \ldots$ satisfying the following conditions:

(1) each of the $f_{\nu}$ is regular except possibly at the supersingular points,

(2) the $f_{\nu}$ restrict one to another: $f_{\nu+1} \equiv f_{\nu}\left(\bmod p^{\nu}\right)$ for all $\nu \geq 1$,

(3) there exist real numbers $C$ and $D$ such that the order of the poles of $f_{\nu}$ at the supersingular points is less than or equal to $C \nu+D$.

The $f_{\nu}$ form an inverse system of functions on the $X_{\mathbb{Z} / p^{\nu} \mathbb{Z}}$ whose poles at the supersingular points have orders that grow at most linearly in $\nu$. We will write

$$
f=\left(f_{\nu}\right)=\lim _{\nu} f_{\nu}
$$

for the overconvergent modular form corresponding to such a family. We define the rate of growth of $f$ to be the greatest lower bound of the $C$ that one can take in the upper bound for the order of the poles.

In the limit, one obtains functions on $X$ which are not defined at points in $X$ corresponding to curves with supersingular reduction. We call the set of points in $X$ whose reductions modulo $p$ are a fixed supersingular point the supersingular disk determined by that supersingular point (it is indeed isomorphic to a $p$-adic open disk in the rigid-analytic sense). Under the hypotheses above, $f$ has a Laurent expansion around each of the supersingular disks which is infinite "to the left", but whose coefficients tend to zero at least linearly.

Consider, for example, what happens for $p=3$ or $p=5$. The only supersingular point is $j=0$, and the Laurent expansion of one of our functions will look like

$$
f=\sum_{n=-0}^{\infty} a_{n} j^{-n} .
$$

For $p=5,1 / j$ has a triple pole at $j=0$, the rate of growth of $f$ will be $\leq C$ if we have

$$
\operatorname{ord}\left(a_{n}\right) \geq \frac{3 n}{C}+k
$$

for some constant $k$ ( $k$ may be negative, but note that we are requiring $\operatorname{ord}\left(a_{n}\right) \geq 0$ in any case). 
Such functions are called overconvergent because they can, in fact, be computed on some curves with supersingular reduction. In fact, if $\varepsilon>0$ and $0<\operatorname{ord}(j)<3 /(C+\varepsilon)$, then

$$
\operatorname{ord}\left(a_{n} j^{-n}\right)>\frac{3 n}{C}-\frac{3 n}{C+\varepsilon}+k
$$

tends to infinity with $n$, and the series converges. In other words, overconvergent functions can be computed on curves that are "not too supersingular."

We extend the definition to $\mathbb{Q}_{p}$ by allowing bounded denominators:

Definition 2. Let $f$ be a function defined on the points of $X$ corresponding to curves without supersingular reduction. We say $f$ is an overconvergent $p$-adic modular function if, for some integer $n \geq 0, p^{n} f$ is an overconvergent $p$-adic modular function with integral $q$-expansion.

Again, one can compute such $f$ at points corresponding to curves which are "not too supersingular," but the values will now be in $\mathbb{Q}_{p}$.

Allowing poles at the supersingular points enlarges our space of functions quite dramatically (it clearly gives an infinite dimensional space). Controlling the rate of growth counteracts this somewhat. It turns out that the optimal choices are when $C$ is around $1+1 / p$ or around $p+1$.

We now specialize to the case when $p=5$. For a given $C$, we write $L(C)$ for the $\mathbb{Z}_{p}$-module of all functions

$$
f=\sum a_{n} j^{-n}
$$

for which the $a_{n}$ are $p$-adic integers which satisfy

$$
\operatorname{ord}\left(a_{n}\right) \geq \frac{3 n}{C}+b(n)
$$

where $b(n) \geq 0$ and $b(n) \rightarrow \infty$ as $n$ grows. We write $B(C)=L(C) \otimes \mathbb{Q}_{p}$, which is an infinite dimensional $\mathbb{Q}_{p}$-vector space. If we put a $p$-adic norm on $B(C)$ by declaring $L(C)$ to be the unit ball, it becomes a $p$-adic Banach space over $\mathbb{Q}_{p}$, which we call the space of overconvergent p-adic modular functions of growth rate $C$.

The usual $q$-expansion map gives an inclusion of $B(C)$ into the space of power series in $q$ with coefficients in $\mathbb{Q}_{p}$. (More precisely, the image is contained in $\mathbb{Q}_{p} \otimes \mathbb{Z}_{p}[[q]]$. In other words, the denominators in such an expansion are bounded.)

It might be worth relating the notion of "growth rate $C$ " to the notation in [6] or [9]. There we measured the degree of overconvergence by a parameter $r$ chosen from an extension $R$ of $\mathbb{Z}_{p}$. The relation is simply that if we choose an $r \in R$ such that $\operatorname{ord}(r)=1 / C$, then the space of 
$r$-overconvergent $p$-adic modular forms of weight 0 is essentially the same as our $B(C)$. Since this is a technical point, we relegate a proof to the appendix.

For every $\ell \neq p$, one can define an action of the Hecke operator $\mathrm{T}_{\ell}$ on $B(C)$. We will call an element of $B(C)$ a Hecke eigenform if it is an eigenfunction of $\mathrm{T}_{\ell}$ for every $\ell \neq p$.

We have found it useful to think of the growth rate $C$ as playing a role something like that of the "the power of $p$ dividing the level" of the overconvergent functions lying in $B(C)$. To pursue this heuristic view a bit, one could denote (for $r \geq 1$ )

$$
B\left(\Gamma_{0}\left(p^{r}\right)\right):=\bigcap_{C>p^{r-2}(p+1)} B(C) \subset \mathbb{Q}_{p} \otimes \mathbb{Z}_{p}[[q]]
$$

and then note that, by [6], any classical modular function for $\Gamma_{0}\left(p^{r}\right)$ with Fourier coefficients in $\mathbb{Q}_{p}$ and with poles only at the ramified cusps lies in $B\left(\Gamma_{0}\left(p^{r}\right)\right)$. Moreover, an analogous statement holds for classical modular forms of arbitrary weight $k$. It would be interesting to establish something of a converse to this, e.g., is it true that a classical modular function for $\Gamma_{0}\left(p^{N}\right)$ with Fourier coefficients in $\mathbb{Q}_{p}$ and with poles only at the ramified cusps which lies in $B\left(\Gamma_{0}\left(p^{r}\right)\right)$ is actually a modular function for $\Gamma_{0}\left(p^{r}\right)$ ? We remind the reader that the spaces $B\left(\Gamma_{0}\left(p^{r}\right)\right)$ are not Banach spaces (at least, they are not naturally so), but they are Frechet spaces with respect to the family of norms inherited from the Banach spaces $B(C)$ for all real numbers $C>p^{r-2}(p+1)$.

\section{The $U$ operator}

Let $B=B(C)$ for some fixed $C>1+p$. For our purposes, the basic operator on $B$ is Atkin's $U$-operator, whose action can be described at the $q$-expansion level by

$$
U: \sum a_{n} q^{n} \longrightarrow \sum a_{n p} q^{n} .
$$

(This description gives no hint about why $U$ maps $B$ to $B$; a proof can be found in [6].) One can show that if $C>1+p$, the $U$ operator decreases the rate of growth. Specifically, $U$ maps functions with growth rate $C$ to functions with growth rate $C / p$. Thus, we get a map

$$
U: B(C) \longrightarrow B(C / p) \hookrightarrow B(C) .
$$

The fact that the $U$ operator factors through the inclusion implies that $U$ is a completely continuous operator. In elementary terms, this means 
the following. Let $L=L(C)$ be the unit ball in $B$. Then, for each $n \geq 0$ there exists a finite dimensional subspace $V_{n} \subset B$ such that

$$
U(L) \subset V_{n}+p^{n} L .
$$

(Essentially, this says that $U$ is the limit of a sequence of operators of finite rank.) Given such an operator, it is natural to ask how the dimension of $V_{n}$ grows as a function of $n$. For the $U$ operator, we gave such an estimate, which we suspect is not optimal, in [9]. One might think of such estimates as measuring the "degree of complete continuity" of an operator.

The spectral theory of completely continuous operators on $p$-adic Banach spaces is analogous to the classical theory due to Riesz and Fredholm. It is systematically developed in [14], to which we refer the reader for details.

Given a rational number $y$, we say a form $f \in B$ is of slope $y$ if there exists a polynomial $G \in \mathbb{Q}_{p}[X]$ such that

- all the roots of $G$ (in $\overline{\mathbb{Q}}_{p}$ ) have valuation $y$, and

- $G(U)(f)=0$.

It follows from the complete continuity that the set of all modular functions of slope $y$ forms a finite dimensional, Hecke equivariant, continuous direct summand of $B$, which we call $B^{y}$. Although the space $B$ depends on our initial choice of the growth rate $C>1+p$, the finite dimensional spaces $B^{y}$ are independent of this choice. There exist continuous projections $e_{y}: B \longrightarrow B^{y}$ which commute with the Hecke operators.

For all $y \geq 0$, the space $B^{y}$ is contained in the Frechet space $B\left(\Gamma_{0}(p)\right)$ of section 1. Now let $B^{[0, \infty]}$ denote the closed subspace of the Frechet space $B\left(\Gamma_{0}(p)\right)$ generated by the spaces $B^{y}$ for all $y$.

It is not known whether the Hecke operators $\mathrm{T}_{\ell}$ can be simultaneously diagonalized on the spaces $B^{y}$ (though it is natural to conjecture that this is the case). It is expected that the action of $U$ on $B^{y}$ may fail to be semisimple, so that the elements of $B^{y}$ may only be generalized eigenfunctions for the $U$ operator. Nevertheless, it is clear that if $B^{y}$ is of dimension one, then any generator will be a simultaneous eigenform for the Hecke and $U$ operators. (The dimension seems to be one in all the examples we computed.) These eigenfunctions give rise, in the standard manner (see [6] or [11]), to two-dimensional, continuous, $p$-adic representations of $\operatorname{Gal}(\overline{\mathbb{Q}} / \mathbb{Q})$ whose determinant is the inverse of the cyclotomic character and whose properties remain rather mysterious.

It follows from Hida's work that if $p \geq 5$ then

$$
\operatorname{dim} B^{0}=\operatorname{dim} M_{p-1}^{0},
$$


where $M_{p-1}^{0}$ denotes the ordinary part of the space of classical eigenforms of weight $p-1$ and level 1 . We have conjectured that a similar result holds in general, that is, that

$$
\operatorname{dim} B^{y}=\operatorname{dim} M_{p^{n}(p-1)}^{y},
$$

where $n$ is any integer greater than or equal to $y$ and the superscript $y$ denotes projection onto the subspace generated by $U$-eigenforms with eigenvalues of valuation $y$. Since the numbers on the right can be computed explicitly (see, for example, [8]), constructing elements of $B^{y}$ provides a mild test of the conjecture.

In [4] and [5], Coleman has proved that there exists an $A$ (depending on $y$ ) such that

$$
\operatorname{dim} B^{y}=\operatorname{dim} M_{p^{n+A}(p-1)}^{y} .
$$

This goes a long way towards proving the conjecture mentioned above, but unfortunately is not helpful from a computational point of view. Given Coleman's result, our computations can be seen as evidence that one can take $A=0$ in our situation, and we conjecture that one can always do so.

Further information on the spectrum of $U$ is hard to come by. We point to two results that may be relevant. First, there is the formula (due to Dwork) that is discussed by Katz in [12, Appendix 3]. This expresses the characteristic power series of the $U$ operator (acting on overconvergent modular functions) as an infinite product of $L$-functions. These $L$ functions are themselves quite mysterious and it seems difficult to obtain much information on the spectrum directly from this result.

A second intriguing result is the recent work of Stevens on "rigidanalytic modular symbols" (see [15]). Stevens constructs, for each weight $k$, a $p$-adic Banach space which extends the classical space of modular symbols attached to modular forms of weight $k$, defines an action of $U$ and of the Hecke operators, and shows that $U$ acts completely continuously. He then shows that the characteristic power series of $U$ acting on this space is related in a simple way to the characteristic power series of $U$ acting on overconvergent $p$-adic modular forms of weight $k$. It seems possible that Stevens' space of modular symbols will prove to be more amenable to computation than the space of overconvergent modular forms.

\section{Spectral decomposition}

Since $U$ is completely continuous, given a prime number $p$ and a tame level $N$, the set of eigenvalues for $U$ acting on the space $B$ of overconvergent functions is a sequence tending to zero. Hence, the set of possible slopes is a sequence of rational numbers (one knows that they are positive rational numbers) tending to infinity. Let $\mathcal{S}=\mathcal{S}(N, p)$ be that sequence. 
For each $i \in \mathcal{S}$, we can consider the projection

$$
e_{i}(f)=\varphi_{i} \in B^{i}
$$

of $f$ to the space of functions of slope $i$. For $0 \leq x \leq \infty$, let $B^{[0, x]}$ denote the subspace of $B$ generated by the eigenspaces $B^{y}$ for $0 \leq y \leq x$. If $x<\infty, B^{[0, x]}$ is finite dimensional, and we have a continuous projection

$$
e_{[x]}(f)=\varphi_{[x]} \in B^{[0, x]} .
$$

We clearly have

$$
\varphi_{[x]}=e_{[x]}(f)=\sum_{i \leq x} e_{i}(f)=\sum_{i \leq x} \varphi_{i}
$$

This suggests that we can associate to $f$ the formal series formed by all the projections to finite slope.

Definition 3. The infinite series

$$
\sum_{i \in \mathcal{S}} e_{i}(f)=\sum_{i \in \mathcal{S}} \varphi_{i}
$$

is called the asymptotic $U$-spectral expansion of $f \in B$.

There is, a priori, no reason to expect this series to converge. One does, however, have the following result, which follows easily from the spectral theory (much as in the complex case):

Proposition 1. Let

$$
\varphi_{[x]}=e_{[x]}(f)=\sum_{i \leq x} e_{i}(f) .
$$

Then there exists $\varepsilon>0$ such that

$$
\operatorname{ord}\left(U^{\nu}\left(f-\varphi_{[x]}\right)\right) \geq \nu(x+\varepsilon) \quad \text { for } \quad \nu \gg 0 .
$$

This justifies using the word "asymptotic" to describe our expansion. It is easy to see that the asymptotic $U$-spectral expansion is the only expansion satisfying the property given by this proposition.

Let $\mathbf{T}$ be the closed algebra of endomorphisms of $B(C)$ generated by the Hecke operators $\mathrm{T}_{\ell}$ for $\ell \neq p$. Since the Hecke operators commute with $U$, the action of $\mathbf{T}$ stabilizes each of the finite dimensional spaces $B^{i}$. It is tempting to try to refine our expansion further by expressing each $\varphi_{i}$ as a linear combination of Hecke eigenfunctions of slope $i$. This would be possible if one knew that the action of $\mathbf{T}$ on each $B^{i}$ is semisimple; unfortunately, one does not know whether that is the case. In the computations below, the functions we obtain do turn out to be Hecke eigenfunctions. 
It is interesting to ask whether the asymptotic $U$-spectral expansion might be a convergent series, and, if so, whether it will converge to $f$ itself. In this direction, note that each of the terms of the expansion are $U$-eigenfunctions (or generalized eigenfunctions) with nonzero eigenvalue, and hence belong to $B\left(\Gamma_{0}(p)\right)$. If the expansion were to converge, one would then expect it to converge to a function in $B\left(\Gamma_{0}(p)\right)$; in fact, it would belong to the subspace denoted $B^{[0, \infty]}$ above. For $f \notin B\left(\Gamma_{0}(p)\right)$, then, the sum of the expansion (if it converges) cannot be equal to $f$. Perhaps one could think of it as a projection of $f$ to $B^{[0, \infty]}$ ?

The computations below exploit first Proposition 1 and then the possibility that the expansions we get are actually convergent.

\section{Some 5-adic modular functions}

Let $p=5$. We attempted to compute subspaces of small slope by starting with a reasonably simple element of $B$ and then iterating the $U$ operator. This is essentially Atkin's idea; see, for example, [1]. (The details of the computations are described in the next section.) The results are consistent with the following picture of $B^{[0,5]}$.

We seem to have $B^{y} \neq 0$ with $y \leq 5$ only for $y=0,1,4$, and 5 . The only classical forms of weight 0 are the constants, which, by Hida's theory, are also the slope 0 subspace. So we set $f_{0}=1$.

The slope 1 subspace seems to be one-dimensional, generated by an eigenform $f_{1}$ such that

$$
\begin{aligned}
f_{1} \equiv & q+8528631 q^{2}+8596652 q^{3}+2788848 q^{4}+3054065 q^{5} \\
& +6727787 q^{6}+2747331 q^{7}+7297460 q^{8}+6989312 q^{9} \\
& +1247515 q^{10}+538817 q^{11}+9643146 q^{12}+6371187 q^{13} \\
& +5536986 q^{14}+7427880 q^{15}+8198461 q^{16}+3226656 q^{17} \\
& +5179372 q^{18}+7613040 q^{19}+8613995 q^{20}+6738937 q^{21} \\
& +275777 q^{22}+658622 q^{23}+3400795 q^{24}+8336725 q^{25} \\
& +2954997 q^{26}+8893915 q^{27}+3799063 q^{28}+6152060 q^{29} \\
& +8960405 q^{30}+5895922 q^{31}+4363161 q^{32}+756309 q^{33} \\
& +3309811 q^{34}\left(\bmod 5^{10}, q^{35}\right)
\end{aligned}
$$

Using the data above, one can check, by computing $\mathrm{T}_{\ell}\left(f_{1}\right)$ directly for prime numbers $\ell$ such that $2 \leq \ell \leq 17$, that $f_{1}$ does behave as an eigenform within the precision of the calculation. For example, $\mathrm{T}_{2}\left(f_{1}\right) \equiv 8528631 f_{1}$ $\left(\bmod 5^{10}, q^{18}\right)$. 
We know the first few coefficients of $f_{1}$ to better precision:

$$
\begin{array}{r}
f_{1} \equiv q+731184881 q^{2}+994924777 q^{3}+246929473 q^{4} \\
+149538440 q^{5}+817274662 q^{6} \quad\left(\bmod 5^{13}, q^{7}\right)
\end{array}
$$

The slope 4 subspace seems also to be one dimensional, generated by an eigenform $f_{4}$ given by

$$
\begin{aligned}
f_{4} \equiv & q+13584 q^{2}+13213 q^{3}+1618 q^{4}+1875 q^{5}+1017 q^{6} \\
& +4844 q^{7}+3370 q^{8}+10452 q^{9}+1250 q^{10}+467 q^{11} \\
& +3634 q^{12}+7143 q^{13}+4021 q^{14}+8750 q^{15} \\
& +11646 q^{16}+1714 q^{17} \quad\left(\bmod 5^{6}, q^{18}\right)
\end{aligned}
$$

(The reason for the loss of precision, both 5-adically and in the number of terms of the $q$-expansion, will become clear when we describe our computation.)

Finally, our computations yield a 5-adic eigenform of slope 5, given by:

$$
\begin{aligned}
f_{5} \equiv & q+14016 q^{2}+8637 q^{3}+2943 q^{4}+9375 q^{5}+9317 q^{6}+156 q^{7} \\
& +7705 q^{8}+9227 q^{9}+9375 q^{10}+2092 q^{11}+12441 q^{12}+2932 q^{13} \\
& +14621 q^{14}+3125 q^{15}+15246 q^{16}+7586 q^{17} \quad\left(\bmod 5^{6}, q^{18}\right)
\end{aligned}
$$

Again, one easily checks that these numbers are consistent with the claim that $f_{4}$ and $f_{5}$ are eigenfunctions.

The conjectures described in [8] and [7] predict that the dimension of the slope 1 subspace in weight 0 should equal the dimension of the slope 1 subspace of the space of classical modular forms of weight 20 and level 1 , and in fact the unique normalized cusp form of level 1 and weight 20 is of slope 1 , and is congruent to $f_{1}$ modulo $5^{2}$.

One can also test the conjectures by considering the subspace of slope 2. In our computations, this is of dimension zero (i.e., we see no forms of slope 2). The conjectures would predict, then, that there are no classical modular forms of slope 2 in weight 100 and level 1, and indeed there are no such forms.

\section{Computing the spectral expansion}

We begin by obtaining a reasonably large number of terms in the $q$ expansion of a 5-adic modular function of tame level 1. To obtain such a form, we can use several different ideas. First, classical modular functions on $\Gamma_{0}\left(5^{n}\right)$ will be overconvergent, and if they are regular at the cusp at infinity then they will be regular from the 5-adic point of view. In particular, we can work with the inverses of the hauptmoduls for levels 5 and 
25 , which are $(\eta(5 z) / \eta(z))^{6}$ and $\eta(25 z) / \eta(z)$. Since the curve with $j=0$ has supersingular reduction, we can also work with $1 / j$. In addition, we can use the $U$ operator to "kill off" a pole; for example, we can work with $U(j)$. Finally, we can take classical modular forms of higher weight $4 k$, and divide them by a power of $\mathrm{E}_{4}$ (which is a lift of the Hasse invariant). In particular, we work with $(\eta(z) \eta(5 z))^{4} / \mathrm{E}_{4}$.

One sees a qualitative difference between the results for functions that are "of level 5" (i.e., that are known to belong to $B\left(\Gamma_{0}(5)\right)$ ) and the results for functions of higher level (e.g., 25). In the first case, the behavior is very close to what one would expect if the asymptotic expansion of $f$ actually converges to $f$. In the second, things are much more fuzzy.

Suppose one has found enough ${ }^{2}$ terms of the $q$-expansion of such a modular function $h$. We can then begin to determine its decomposition. We describe the process in a "good" case, i.e., a form that belongs to $B\left(\Gamma_{0}(5)\right)$.

First, we subtract off any constant term in the $q$-expansion, which will be the projection to slope zero. Let $c_{0}$ be the constant term, and set $h^{\prime}=h-c_{0}$. We know, then, that $U^{n}\left(h^{\prime}\right)$ will be divisible by $5^{n}$ when $n$ is sufficiently large. Optimistically, we assume this will hold for all $n \geq 1$ (as indeed it does in our computations, for functions of level 5 , at least for the first several terms in the $q$-expansion ${ }^{3}$ ), and we compute $h_{1}=U\left(h^{\prime}\right) / 5$, and $h_{2}=U\left(h_{1}\right) / 5$.

If we assume that

$$
h=c_{0}+c_{1} f_{1}+\text { terms of higher slope, }
$$

we would expect that $h_{2}$ should be congruent to a multiple of $c_{1} f_{1}$ modulo a high power of 5 , and should therefore be highly congruent to a Hecke eigenform. We check this by dividing $h_{2}$ by its leading coefficient to get a normalized form $h_{2}^{\prime}$, computing $T_{2}\left(h_{2}^{\prime}\right)-a_{2} h_{2}^{\prime}$ (where $a_{2}$ is the coefficient of $q^{2}$ in the $q$-expansion of $\left.h_{2}^{\prime}\right)$ and checking the 5 -adic valuations of all the coefficients we have available. If $m$ is the smallest of these valuations, we reduce modulo $5^{m}$. In all of our computations, this process always produces (to the precision available) the same form $f_{1}$, and we check that it is a Hecke eigenform (to the precision available). Both facts strongly suggest that the subspace of slope 1 functions is one-dimensional.

To compute the coefficient $c_{1}$, we must divide the leading coefficient of $h_{2}$ by $(\lambda / 5)^{2}$, where $\lambda$ is the eigenvalue of $U$ on $f_{1}$. To obtain this as

\footnotetext{
${ }^{2}$ How much is enough depends on how many terms of the $q$-expansion we want to test at the end, and to what 5-adic precision. When we could, we used over a thousand terms.

${ }^{3}$ For the case $h=U(j)$, the results announced by Atkin in [1] would imply that this does hold.
} 
precisely as possible, we took the best of our computations (for $h=1 / j$ ) and computed $h_{3}=U\left(h_{2}\right) / 5$, which gives us (fewer terms of) $f_{1}$ modulo $5^{13}$, so that in particular we know $\lambda / 5$ modulo $5^{12}$, which allows us to compute $c_{1}$ without loosing any 5 -adic precision.

Now we look at $h^{\prime \prime}=h-c_{0}-c_{1} f_{1}$. We expect that $U\left(h^{\prime \prime}\right)$ should be highly divisible by 5 (though the theorem only assures us of this for high powers of $U$ ). Computing $U$ more times, however, would require us to know many terms of the $q$-expansion of $f_{1}$, which would require much too many terms in the initial expansion of $h$. So we proceed in a different way, which depends on the possibility that the asymptotic expansion actually approximates $h$.

We first test $h^{\prime \prime}$ for divisibility by 5 ; it is quite often divisible by $5^{3}$ (all but one of the computations). After dividing by a power of 5 (and therefore losing some 5-adic precision), we attempt to diagonalize the action of the Hecke operators on the space generated by $h^{\prime \prime}$. In practice, we diagonalize the action of $T_{2}$ on the space spanned by $h^{\prime \prime}$ and $T_{2}\left(h^{\prime \prime}\right)$ (checking that this space is Hecke-stable modulo a power of 5), and check that the resulting two functions are Hecke eigenfunctions (within the limits of our precision, both 5 -adic and in $q$-expansion). In all our computations, these functions are $f_{4}$ and $f_{5}$. The actual slopes are determined in the computation for $1 / j$, which allows us to find both functions modulo $5^{6}$. The fact that this computation succeeds (and gives always the same two eigenfunctions $f_{4}$ and $f_{5}$ ) is evidence that the spaces of slopes 4 and 5 are both onedimensional. It also suggests that the next step in the decomposition should involve functions of somewhat higher slope.

The result is an asymptotic expansion

$$
h \sim c_{0}+c_{1} f_{1}+c_{4} f_{4}+c_{5} f_{5}+\ldots \quad\left(\bmod 5^{m}, q^{n}\right)
$$

In Table 2, we record the coefficients obtained for various choices of $h$. In addition, we record the 5 -adic precision $m$ and the number $n$ of terms of the $q$-expansion which were checked.

We only did two computations for functions which are not known to be "of level 5" in the sense introduced earlier. These were $g_{1}=\eta(25 z) / \eta(z)$, which is the inverse of a hauptmodul for level 25, and the form $g_{2}=$ $(\eta(5 z) \eta(25 z))^{4} / \mathrm{E}_{4}$, which is an oldform of weight 4 and level 25 divided by $\mathrm{E}_{4}$. For both of these functions, the first half of the computation works well, giving the same form $f_{1}$ of slope 1 modulo a power of 5 . (There is one slight glitch for $g_{2}$. In this case $U^{3}\left(g_{2}\right)$ is only divisible by $5^{2}$, but dividing by $5^{2}$ yields a multiple of $f_{1}$ modulo $5^{10}$.)

In both cases, however, the second part of the process does not work as well. For $g_{1}$, the expression we get for $\left(T_{2}\right)^{2}\left(h^{\prime \prime}\right)$ (using the notation above) as a linear combination of $h^{\prime \prime}$ and $T_{2}\left(h^{\prime \prime}\right)$ is only correct modulo $5^{3}$, 


\begin{tabular}{|c||c|c|c|c||c|c|}
\hline Function & $c_{0}$ & $c_{1}$ & $c_{4}$ & $c_{5}$ & $n$ & $m$ \\
\hline $1 / j$ & 0 & 8295001 & $5^{4} \cdot 5786$ & $5^{4} \cdot 12192$ & 18 & 10 \\
\hline$\frac{(\eta(z) \eta(5 z))^{4}}{\mathrm{E}_{4}}$ & 0 & 1121501 & $5^{4} \cdot 1031$ & $5^{3} \cdot 1498$ & 17 & 9 \\
\hline$U(j)$ & 744 & $5^{2} \cdot 14624$ & $5^{5} \cdot 93$ & $5^{6} \cdot 2$ & 17 & 8 \\
\hline$\left(\frac{\eta(5 z)}{\eta(z)}\right)^{6}$ & 0 & 559751 & $5^{3} \cdot 532$ & $5^{4} \cdot 248$ & 17 & 8 \\
\hline$\frac{\eta(25 z)}{\eta(z)}$ & 0 & 4377 & $*$ & $*$ & 35 & 6 \\
\hline$\frac{(\eta(5 z) \eta(25 z))^{4}}{\mathrm{E}_{4}}$ & 0 & $5^{-1} \cdot 244252$ & $*$ & $*$ & 25 & 10 \\
\hline
\end{tabular}

TABLE 2. $U$-spectral expansion of modular functions

and one only recovers $f_{4}$ and $f_{5}$ to a very low 5 -adic accuracy (modulo 5 in one case, modulo $5^{2}$ in the other). For $g_{2}$, the fact that $U^{3}\left(g_{2}\right)$ is only divisible by $5^{2}$ makes the coefficient $c_{1}$ have 5 -adic valuation -1 , and this makes the rest of the computation hard to carry out. In the table, we have recorded only the results of the first half of the computation.

Notice that this behavior is consistent with the fact that the "error term" (the difference between the initial form and a partial sum of the asymptotic expansion) must be "more serious" in this case than in the level 5 case, since all the terms of the asymptotic expansion are of level 5. In the level 5 case, it is conceivable that the asymptotic expansion is actually convergent, while in the general case it cannot be. In fact one might ask ...

Question 1. Suppose $f \in B\left(\Gamma_{0}(p)\right)$. Does the $U$-spectral expansion of $f$ converge to $f$ ?

Notes on the table:

(1) The coefficients $c_{0}$ are in fact known to infinite $p$-adic precision, since they are simply the constant terms in the $q$-expansions.

(2) For the "level 5" computations (the first four lines), the initial portion of the expansion, $c_{0}+c_{1} f_{1}$, was in fact computed to $2 n$ terms of the $q$-expansion; this precision was halved in the second part of the computation by computing $T_{2}$. 
(3) The verification that the functions that appear in the expansions are indeed $f_{1}, f_{4}$, and $f_{5}$ is done to the precision allowed by the data. Hence, for example, the identification of $f_{1}$ was checked modulo $5^{10}$ for the first row, but only modulo $5^{6}$ for the third. In particular, when $h=U(j) / 25$, we can only identify $f_{4}$ modulo $5^{3}$, and $f_{5}$ modulo $5^{2}$.

(4) In the fourth line, $c_{1}$ is in fact known modulo $5^{9}$, but finding the other two coefficients involves a division by 5 .

(5) In the last two lines, which correspond to functions which are not "of level 5", we have recorded only the coefficient which we can determine to some degree of precision, namely, $c_{1}$.

(6) Note that for the "level 5" cases the coefficients $c_{4}$ and $c_{5}$ seem to be highly divisible by 5 . This is consistent with a positive answer to our question about whether the $U$-spectral expansion of a form "of level 5 " is actually convergent.

\section{Some 3-adic results}

We have done similar computations in a 3 -adic context. In this case, one can use results of Hatada [10], together with the conjectures in [8] and [7], to predict that the smallest nonzero slope is 2. Our findings are consistent with the following picture for the space $B^{[0,6]}$ :

- $B^{y}$ is nonzero only for $y=0,2$, and 6 .

- $B^{0}$ is one-dimensional, generated by $f_{0}=1$.

- $B^{6}$ is one-dimensional, generated by an eigenform

$$
\begin{aligned}
f_{6} \equiv & q+8088 q^{2}+14580 q^{3}+18976 q^{4}+4818 q^{5}+2187 q^{6} \\
& +14801 q^{7}+5493 q^{8}+15327 q^{10}+13128 q^{11}+5832 q^{12} \\
& +7073 q^{13}+18165 q^{14}+17496 q^{15}+13048 q^{16}+14400 q^{17} \\
& +18047 q^{19}+18516 q^{20}+13851 q^{21}+9162 q^{22}+4908 q^{23} \\
& +17496 q^{24}+14740 q^{25}+7626 q^{26}+7049 q^{28}+17619 q^{29} \\
& +6561 q^{30}+15344 q^{31}+18756 q^{32}+8748 q^{33} \\
& +2889 q^{34}+19392 q^{35}\left(\bmod 3^{10}, q^{36}\right)
\end{aligned}
$$


- $B^{2}$ is one-dimensional, generated by an eigenform

$$
\begin{aligned}
f_{2} \equiv & +228432 q^{2}+2136060 q^{3}+1378318 q^{4}+2704551 q^{5} \\
& +309447 q^{6}+3835247 q^{7}+3322797 q^{8}+782298 q^{9} \\
& +4237209 q^{10}+3299286 q^{11}+3813192 q^{12}+4463207 q^{13} \\
& +1493943 q^{14}+2800224 q^{15}+3992659 q^{16}+148653 q^{17} \\
& +608958 q^{18}+1760357 q^{19}+77874 q^{20}+573930 q^{21} \\
& +508284 q^{22}+3870618 q^{23}+2129301 q^{24}+663307 q^{25} \\
& +1629384 q^{26}+20412 q^{27}+23087 q^{28}+4523646 q^{29} \\
& +843615 q^{30}+4221431 q^{31}+1801071 q^{32}+2397141 q^{33} \\
& +2805165 q^{34}+1906464 q^{35}+2015280 q^{36}+1687682 q^{37} \\
& +3317487 q^{38}+1070325 q^{39}+1324737 q^{40}+3992676 q^{41} \\
& +2797470 q^{42}+3528329 q^{43}+108570 q^{44}+4152141 q^{45} \\
& +2927574 q^{46}+3124254 q^{47}+4112919 q^{48}+322806 q^{49} \\
& +869673 q^{50}+4764177 q^{51}+2524127 q^{52}+2754459 q^{53} \\
& +4142178 q^{54}+4199031 q^{55}+4622259 q^{56}+1434690 q^{57} \\
& +4182498 q^{58}+2860773 q^{59}+1440558 q^{60}+2625458 q^{61} \\
& +1197195 q^{62}+2216565 q^{63}+1218385 q^{64}+770997 q^{65} \\
& +723978 q^{66}+1331693 q^{67}+3062601 q^{68}+3373866 q^{69} \\
& +3274029 q^{70} \quad\left(\bmod 3^{14}, q^{71}\right)
\end{aligned}
$$

- Finally, we find one more eigenform, determined $\left(\bmod 3^{6}, q^{36}\right)$, which is of slope bigger than 6 .

\section{Appendix: relating the two definitions}

The definition of overconvergent forms and of the Banach spaces $B(C)$ given in section 1 is different from the one found in [12] or in [6]. The purpose of this section is to reconcile the two definitions by showing that they are in fact equivalent. We begin by recalling the basic definitions. It seems more natural to put this more generally at first, and work with general weight $k$ and level $N$.

Let $p$ be a prime number, $p \geq 5$, let $R$ denote the ring of integers in a finite extension $K$ of $\mathbb{Q}_{p}$, and let $\mathrm{E}_{p-1}$ be the classical Eisenstein series of weight $p-1$. About $\mathrm{E}_{p-1}$, recall that it can be understood as a function 
of an elliptic curve with a non-vanishing differential, and that $\mathrm{E}_{p-1}(E, \omega)$ $(\bmod p)$ is equal to the Hasse invariant of the reduction of $E$.

Definition 4. Let $N$ be an integer not divisible by $p$, and let $r \in R$. A test-object of level $N$ and growth condition $r$ over $R$ is a quadruple $(E / A, \omega, \iota, Y)$, where

(1) $E$ is an elliptic curve over a $p$-adically complete and separated $R$-algebra $A$,

(2) $\omega$ is a non-vanishing differential on $E$,

(3) $\iota: \boldsymbol{\mu}_{N} \hookrightarrow E[N]$ is an inclusion of finite flat group-schemes over $A$ and

(4) $Y \in A$ satisfies the equation

$$
Y \cdot \mathrm{E}_{p-1}(E, \omega)=r,
$$

where $\mathrm{E}_{p-1}$ is the classical Eisenstein series of weight $p-1$.

The idea is to mimic the classical definition of an elliptic curve with level structure, but add a constraint on ord $\left(\mathrm{E}_{p-1}(E, \omega)\right)$. Now we define $p$-adic modular forms as functions of test-objects.

Definition 5. Let $r \in R$, and let $N$ and $k$ be integers, with $p \nmid N$. An $r$-overconvergent $p$-adic modular form of weight $k$ and level $N$ defined over $R$ is a rule $f$ which assigns to each test-object $(E / A, \omega, \iota, Y)$ of level $N$ and growth condition $r$ defined over $R$, an element

$$
f(E / A, \omega, \iota, Y) \in A,
$$

called the value of $f$ at the test-object, satisfying the following conditions:

(1) The value $f(E / A, \omega, \iota, Y)$ depends only on the isomorphism class of the test-object $(E / A, \omega, \iota, Y)$.

(2) The formation of the value $f(E / A, \omega, \iota, Y)$ commutes with basechange of $p$-adically complete $R$-algebras.

(3) For any $\lambda \in A^{\times}$we have

$$
f(E / A, \lambda \omega, \iota, Y)=\lambda^{-k} f(E / A, \omega, \iota, Y) .
$$

(4) Let Tate $(q)$ be the Tate elliptic curve over $\mathbb{Z}_{p}((q))$, and consider it as an elliptic curve over the $p$-adic ring $A=R \hat{\otimes} \mathbb{Z}_{p}((q))$ of Laurent series in $q$ with coefficients in $R$. Let $A_{0}$ denote the subring of power series in $q$ :

$$
A_{0}=R \hat{\otimes} \mathbb{Z}_{p}[[q]]=R[[q]] \subset A=R \hat{\otimes} \mathbb{Z}_{p}((q)) .
$$


Let $\omega$ be the canonical differential on $\operatorname{Tate}(q)$, and let $\iota$ be any inclusion $\iota: \boldsymbol{\mu}_{N} \hookrightarrow \operatorname{Tate}(q)[N]$ of group schemes over $A$. Finally, recall that $\mathrm{E}_{p-1}(\operatorname{Tate}(q))$ is invertible in $\mathbb{Z}_{p}[[q]]$, and let $Y=r \mathrm{E}_{p-1}(\operatorname{Tate}(q), \omega)^{-1}$. Then we require that

$$
f\left(\operatorname{Tate}(q){ }_{A}, \omega, \iota, Y\right) \in A_{0}=R[[q]] .
$$

Condition (4) is to be understood as requiring "holomorphicity at the cusps." This is because the Tate curve with various level $N$ structures gives uniformizations of formal neighborhoods of the cusps.

Definition 6. We denote by $M_{k}(R, N ; r)$ the $R$-module of all $p$-adic modular forms of weight $k$, level $N$, and growth condition $r$ which are defined over $R$, and we define $M_{k}(K, N ; r)=M_{k}(R, N ; r) \otimes K$.

If we give the $K$-vector space $M_{k}(K, N ; r)$ the $p$-adic metric whose unit ball is equal to $M_{k}(R, N ; r)$, it becomes a $p$-adic Banach space over $K$. If $r_{1} \mid r_{2}$, there is a map

$$
M_{k}\left(K, N ; r_{2}\right) \longrightarrow M_{k}\left(K, N ; r_{1}\right),
$$

which can be shown to be an inclusion. When $\operatorname{ord}\left(r_{2}\right)>\operatorname{ord}\left(r_{1}\right)$ this map is completely continuous; when $\operatorname{ord}\left(r_{1}\right)=\operatorname{ord}\left(r_{2}\right)$, it is an isomorphism. Using this, we can think of all of our spaces as contained in $M_{k}(K, N ; 1)$, which we shall call the space of all $p$-adic modular forms of weight $k$ and level $N$ (over $K$ ).

We want to say a $p$-adic modular form is overconvergent if it belongs to $M_{k}(K, N ; r)$ for a non-unit $r$. We have to be just a little careful because of the requirement that $r \in R$, and we will call the notion we are defining "M-overconvergence" to distinguish it from the notion used in the main text.

Definition 7. A $p$-adic modular form $f \in M_{k}(K, N ; 1)$ is said to be Moverconvergent if there exists a finite extension $L$ of $K$ and a non-unit element $r$ in the ring of integers of $L$ such that $f \in M_{k}(L, N ; r)$.

The goal of this appendix is to prove two facts:

(1) a modular function is $M$-overconvergent if and only if it is overconvergent (in the sense of Definition 1);

(2) if $r \in K$ is such that $\operatorname{ord}(r)=1 / C$, then $M_{0}(K, N ; r)$ is isomorphic to the space $B(C)$.

The main tool to establish this connection is a more explicit description of $p$-adic modular forms given by Katz in [12], as sums of power series in $\mathrm{E}_{p-1}^{-1}$. We recall how that is done, specializing to the case of modular functions, i.e., modular forms of weight zero. 
Given $k$ and $N$, let $M_{k}^{c l}(N)$ denote the space of classical modular forms of weight $k$ on $\Gamma_{1}(N)$ with coefficients in $\mathbb{Z}_{p}$. For each $k$, multiplication by $\mathrm{E}_{p-1}$ gives an injective map

$$
M_{k}^{c l}(N) \longrightarrow M_{k+p-1}^{c l}(N) \text {. }
$$

Katz shows that this map is split, and hence that for every $i \geq 0$ we can choose a submodule $B_{i}\left(\mathbb{Z}_{p}, N\right) \subset M_{i(p-1)}^{c l}(N)$ such that

$$
M_{i(p-1)}^{c l}(N)=\mathrm{E}_{p-1} M_{(i-1)(p-1)}^{c l}(N) \oplus B_{i}\left(\mathbb{Z}_{p}, N\right) .
$$

(For $i=0$, we just take $B_{0}\left(\mathbb{Z}_{p}, N\right)=M_{0}^{c l}(N)=\mathbb{Z}_{p}$.) We extend this to general $\mathbb{Z}_{p}$-algebras $R$ by setting $B_{i}(R, N)=B_{i}\left(\mathbb{Z}_{p}, N\right) \otimes R$. For $f \in$ $B_{i}(R, N)$ with $q$-expansion $\sum a_{n} q^{n}$, we set ord $(f)=\inf \operatorname{ord}_{p}\left(a_{n}\right)$, where as usual we normalize the $p$-adic ord by requiring $\operatorname{ord}_{p}(p)=1$. It follows from the $q$-expansion principle for classical modular forms that $\operatorname{ord}(f)=x>0$ implies that $f=s f_{0}$, where $s \in R, \operatorname{ord}(s)=x$ and $\operatorname{ord}\left(f_{0}\right)=0$.

With these notations, Katz shows that any element of $M_{0}(R, N ; 1)$ can be written as

$$
\sum_{i=0}^{\infty} b_{i} \mathrm{E}_{p-1}^{-i},
$$

where $b_{i} \in B_{i}(R, N)$ and we have ord $\left(b_{i}\right) \rightarrow \infty$. Furthermore, we can show that $f \in M_{k}(R, N ; r)$ if and only if we have

- $\operatorname{ord}\left(b_{i}\right) \geq i \operatorname{ord}(r)$ for every $i$

- $\operatorname{ord}\left(b_{i}\right)-i \operatorname{ord}(r) \rightarrow \infty$ as $i \rightarrow \infty$.

(If we only want to identify which $f \in M_{k}(K, N ; r)$, then the second condition will suffice.)

We are now ready to prove that "overconvergent" (as defined in the text) and "M-overconvergent" are equivalent. We formulate our argument in the specific context of weight zero and level 1, but a similar argument would work in a more general setting.

Proposition 2. Let $R$ be a finite extension of $\mathbb{Z}_{p}$, and let $r \in R$ be a non-unit. Let $f \in M_{0}(R, 1 ; r)$. Then there exist rational functions $f_{\nu}$ on $X_{\mathbb{Z} / p^{\nu} \mathbb{Z}}=X \otimes \mathbb{Z} / p^{\nu} \mathbb{Z}$ for $\nu=1,2, \ldots$ satisfying the following conditions:

(1) each of the $f_{\nu}$ is regular except possibly at the supersingular points;

(2) the $f_{\nu}$ restrict one to another: $f_{\nu+1} \equiv f_{\nu}\left(\bmod p^{\nu}\right)$ for all $\nu \geq 1$;

(3) there exist constants $C$ and $D$ such that the order of the poles of $f_{\nu}$ at the supersingular points is less than or equal to $C \nu+D$;

(4) the $f_{\nu}$ converge to $f$ in the q-expansion topology.

In other words, if $f \in M_{0}(R, 1 ; r)$, then $f$ is overconvergent with integral q-expansion. 
Proof. Suppose $f \in M_{0}(R, 1 ; r)$. Then, as above, we can write

$$
f=\sum_{i=0}^{\infty} r^{i} b_{i} \mathrm{E}_{p-1}^{-i}
$$

with $b_{i} \in B_{i}(R, 1)$. Given $\nu$, let

$$
a=\left\lceil\frac{\nu}{\operatorname{ord}(r)}\right\rceil
$$

be the smallest integer that is greater than or equal to $\nu / \operatorname{ord}(r)$. Then

$$
f \equiv \sum_{i=0}^{a} r^{i} b_{i} \mathrm{E}_{p-1}^{-i}=\frac{1}{\mathrm{E}_{p-1}^{a}} \sum_{i=0}^{a} r^{i} b_{i} \mathrm{E}_{p-1}^{a-i} \quad\left(\bmod p^{\nu}\right),
$$

and the right-hand side is a rational function on $X_{\mathbb{Z} / p^{\nu} \mathbb{Z}}$ with poles of order at most $a$ at the supersingular points. Hence, if we set

$$
f_{\nu}=\frac{1}{\mathrm{E}_{p-1}^{a}} \sum_{i=0}^{a} r^{i} b_{i} \mathrm{E}_{p-1}^{a-i},
$$

we find that $f_{\nu}$ has a pole of order at most

$$
\left\lceil\frac{\nu}{\operatorname{ord}(r)}\right\rceil<\frac{n}{\operatorname{ord}(r)}+1
$$

This gives the system of rational functions $f_{\nu}$ we are looking for, with $C=1 / \operatorname{ord}(r)$ and $D=1$.

The converse is just a little harder.

Proposition 3. Let $f$ be an overconvergent modular function with integral $q$-expansion. Then $f$ is $M$-overconvergent.

Proof. Suppose $f$ is an overconvergent modular function with integral $q$ expansion, so that we have a system of rational functions $f_{\nu}$ as above. Enlarging $C$ if necessary, we can assume that $f_{\nu}$ has poles of order at most $C \nu$ at the supersingular points. Then $\mathrm{E}_{p-1}^{C \nu} f_{\nu}$ will have no poles at the supersingular points, and hence will be a classical modular form of weight $C \nu(p-1)$. Using Katz's result above, we can write this in terms of the $B_{i}\left(\mathbb{Z}_{p}, 1\right)$, so that

$$
\mathrm{E}_{p-1}^{C \nu} f_{\nu}=\sum_{i=0}^{C \nu} b_{i}^{(\nu)} \mathrm{E}_{p-1}^{C \nu-i}
$$

and therefore

$$
f_{\nu}=\sum_{i=0}^{C \nu} b_{i}^{(\nu)} \mathrm{E}_{p-1}^{-i}
$$


with $b_{i} \in B_{i}\left(\mathbb{Z}_{p}, 1\right)$.

Now, for every $\nu$ we know that $f_{\nu}-f_{\nu-1}$ is divisible by $p^{\nu-1}$. This translates to congruences for the $b_{i}^{(\nu)}$ :

$$
\begin{cases}b_{i}^{(\nu)}-b_{i}^{(\nu-1)} \equiv 0\left(\bmod p^{\nu-1}\right), & \text { for } i=0,1, \ldots C(\nu-1) \\ b_{i}^{(\nu)} \equiv 0\left(\bmod p^{\nu-1}\right), & \text { for } i>C(\nu-1)\end{cases}
$$

It follows that for each $i$ the sequence $b_{i}^{(\nu)}$ converges in $B_{i}\left(\mathbb{Z}_{p}, 1\right)$, so that we may set

$$
b_{i}=\lim _{\nu \rightarrow \infty} b_{i}^{(\nu)} \in B_{i}\left(\mathbb{Z}_{p}, 1\right),
$$

and we note that we also get that for each $\nu \geq 1$

$$
\operatorname{ord}\left(b_{i}\right) \geq \nu-1 \quad \text { if } i>C(\nu-1) .
$$

This implies that the $b_{i}$ "tend to zero", so that we may define

$$
f=\sum_{i=0}^{\infty} b_{i} \mathrm{E}_{p-1}^{-i}
$$

We clearly have $f_{\nu} \rightarrow f$ as $\nu \rightarrow \infty$, and it remains to check that $f$ is M-overconvergent.

To see this, let $R$ be a finite extension of $\mathbb{Z}_{p}$ in which there exists an element $r$ satisfying $\operatorname{ord}(r)=1 / 2 C$. Then we have

$$
\operatorname{ord}\left(r^{C} b_{i}\right) \geq \nu-\frac{1}{2} \quad \text { if } \quad i>C(\nu-1)
$$

which gives

$$
\operatorname{ord}\left(r^{C} b_{i}\right) \geq i \operatorname{ord}(r) \text { for all } i
$$

and

$$
\operatorname{ord}\left(r^{C} b_{i}\right)-i \operatorname{ord}(r) \rightarrow \infty \quad \text { as } i \rightarrow \infty,
$$

which means that $r^{C} f \in M_{0}(R, 1 ; r)$, so that $f$ is M-overconvergent, as claimed.

In fact, it is easy to see that the argument at the end may be modified to show that for any $r$ such that $\operatorname{ord}(r)<1 / C$, some multiple of $f$ is $r$-overconvergent.

Corollary 4. "Overconvergent" and "M-overconvergent" are equivalent. 
Now it remains to consider the explicit definition of the spaces $L(C)$ and $B(C)$ given in the text. Since the definition uses the fact that one has an expansion in powers of $j^{-1}$, it depends specifically on the fact that we are working with $p=5$, weight zero, and level 1 . Restricting to this situation allows us to describe the spaces $B_{i}\left(\mathbb{Z}_{p}, 1\right)$ completely explicitly, since we know that the algebra of all classical forms is generated by $\mathrm{E}_{p-1}=\mathrm{E}_{4}$ and $\mathrm{E}_{6}$. We can take:

- $B_{0}(1) \cong \mathbb{Z}_{p}$,

- if $i=3 k$, then $B_{i}\left(\mathbb{Z}_{p}, 1\right)=\mathrm{E}_{6}^{2 k} \mathbb{Z}_{p}$,

- otherwise, $B_{i}\left(\mathbb{Z}_{p}, 1\right)=0$.

Thus, the expansion of a typical $f \in M_{0}(R, 1 ; r)$ will look like

$$
f=\sum_{k=0}^{\infty} \frac{r^{3 k} a_{k} \mathrm{E}_{6}^{2 k}}{\mathrm{E}_{4}^{3 k}}=\sum_{k=0}^{\infty} r^{3 k} a_{k}\left(\frac{\mathrm{E}_{6}^{2}}{\mathrm{E}_{4}^{3}}\right)^{k}
$$

with $a_{k} \in R, a_{k} \rightarrow 0$.

Furthermore, one can easily find the expansion of $1 / j$ :

$$
\frac{1}{j}=\frac{\mathrm{E}_{4}^{3}-\mathrm{E}_{6}^{2}}{1728 \mathrm{E}_{4}^{3}}=\frac{1}{1728}\left(1-\frac{\mathrm{E}_{6}^{2}}{\mathrm{E}_{4}^{3}}\right),
$$

so that, in particular, $r^{3} j^{-1} \in M_{0}(R, 1 ; r)$ (for any $r$ ). Conversely, we have

$$
\frac{\mathrm{E}_{6}^{2}}{\mathrm{E}_{4}^{3}}=1+\frac{1728}{j}
$$

and these two formulas give an easy way to translate between our expansions and the expansions in $1 / j$ used in the text, giving:

Proposition 5. Let $R$ be a finite extension of $\mathbb{Z}_{p}$, let $K$ be its field of fractions, and let $r \in R$ be an element with $\operatorname{ord}(r)=1 / C$. Then we have

$$
L(C) \hat{\otimes} R=M_{k}(R, 1 ; r)
$$

and

$$
B(C) \hat{\otimes} K=M_{k}(K, 1 ; r) .
$$

Proof. Just use equations (1) and (2) to change from an expansion in terms of $\mathrm{E}_{6}^{2} / \mathrm{E}_{4}^{3}$ to one in $j^{-1}$ and vice-versa. 


\section{References}

1. A. O. L. Atkin, Congruences for modular forms, Computers in Mathematical Research, R. F. Churchhouse and J. C. Herz, eds., North-Holland, Amsterdam, 1968.

2. _ Congruence Hecke operators, Number Theory, W. J. Leveque and E. G. Strauss, eds., Proceedings of Symposia in Pure Mathematics, vol. XII, AMS, Providence, 1969, pp. 33-40.

3. A. O. L. Atkin and J. N. O'Brien, Some properties of $p(n)$ and $c(n)$ modulo powers of 13, Trans. Amer. Math. Soc. 126 (1967), 442-459.

4. R. F. Coleman, Classical and overconvergent modular forms, preprint.

5. , p-adic Banach spaces and families of modular forms, preprint.

6. F. Q. Gouvêa, Arithmetic of p-adic modular forms, Lecture Notes in Mathematics, vol. 1304, Springer-Verlag, New York, 1988.

7. C Continuity properties of p-adic modular forms, Elliptic Curves and Related Topics, H. Kisilevsky and M. R. Murty, eds., CRM Proceedings and Lecture Notes, vol. 4, AMS, 1994, pp. 85-99.

8. F. Q. Gouvêa and B. Mazur, Families of modular eigenforms, Math. Comp. 58 (1992), 793-806.

9. $\quad$ On the characteristic power series of the U operator, Ann. Inst. Fourier (Grenoble) 43 (1993), 301-312.

10. K. Hatada, Eigenvalues of Hecke operators on $\mathrm{SL}_{2}(\digamma)$, Math. Ann. 239 (1979), 75-96.

11. H. Hida, Theory of p-adic Hecke algebras and Galois representations, Sugaku Expositions 2 (1989), 75-102.

12. N. M. Katz, p-adic properties of modular schemes and modular forms, Modular Forms in One Variable III (SLN 350), W. Kuijk and Jean-Pierre Serre, eds., Springer-Verlag, New York, 1973.

13. M. Koike, On some p-adic properties of the Eichler-Selberg trace formula, Nagoya Math. J. 56 (1974), 45-52.

14. J. P. Serre, Endomorphismes complètement continus des espaces de Banach padiques, Publ. Math. I.H.E.S. 12 (1962), 69-85.

15. G. Stevens, Rigid-analytic modular symbols and families of modular forms, preprint, 1994.

Department of Mathematics and Computer Science, Colby College, WaterVILLE ME 04901

E-mail address: fqgouvea@colby.edu

Department of Mathematics, Harvard University, One Oxford St., CamBRIDGE MA 02138

E-mail address: mazur@math.harvard.edu 